\title{
Evaluation of the Physical Stability of Nanostructured Lipid Carrier (NLC) Meloxicam Before and After Strorage 40 Days
}

\author{
Rahmi Annisa $^{1}$, Dewi Melani ${ }^{2}$, Esti Hendradi ${ }^{2}$ \\ ${ }^{1}$ Pharmacy Department of Faculty of Medicine and Health Sciences, Universitas Islam Negeri Maulana Malik Ibrahim, \\ Malang, East Java, Indonesia \\ ${ }^{2}$ Pharmaceutics Department of Faculty of Pharmacy, Universitas Airlangga, Surabaya, East Java, Indonesia.
}

Received: $26^{\text {th }}$ Oct, 17; Revised: $7^{\text {th }}$ Dec, 17, Accepted: $13^{\text {th }}$ May, 18; Available Online: $25^{\text {th }}$ Jun, 2018

\begin{abstract}
Objective: The aims this study was to determine the effect of lipid ratio Monostearin and Miglyol 808 roomates gives the physical stability after storage 40 days. Methods: The NLC making was done by using emulsification method in the formation of NLC meloxicam, 3 different lipid ratios were used, including ratios of 6: 4, 7:3, 8: 2. Results: NLC meloxicam physical stability of the $\mathrm{pH}$, viscosity, particle size, particle morphology and entrapment efficiency. NLC meloxicam belongs to semisolid preparations with $\mathrm{pH}$ value range of 5.72-5.87. Increasing viscosity of NLC system is caused by the increase of solid lipid. The measurement results of particle size of three different lipid formulas indicated that the lipid particle size was $<1000 \mathrm{~nm}$. Entrapment efficiency test of all NLC-lipid compositions revealed quite high result (> $80 \%$ ). Conclusion: The system uses lipid monostearin NLC meloxicam and Miglyol 808 at a ratio of 8: 2 is the most optimal combination shows physical stability after storage 40 days.
\end{abstract}

Keywords: Nanostructured Lipid Carriers, NLC, Meloxicam, physical stability.

\section{INTRODUCTION}

Meloxicam belongs to a group of Non-Steroidal AntiInflammatory Drugs. Meloxicam can be used in rheumathoid arthritis therapy. However, the use of oral meloxicam causes gastrointestinal irritation side effects ${ }^{1}$. The topical use of meloxicam can be delivery solutions of NSAID drug, directly to the target disease and provide a local effect. This method can also avoid the existence of gastrointestinal side effects because it is not absorbed systemically. Meloxicam has a characteristic which is not soluble in water and has a $\log P$ value of $3: 42$, so in a preparation mostly consisting of water, the solubility of meloxicam would be very small and with the $\log \mathrm{P}$ values, it means that meloxicam is non-polar. Therefore, the development of this meloxicam topical delivery systems. One of delivery system was selected is Solid Lipid Nanoparticle

(SLN).

Solid Lipid Nanoparticle (SLN) is colloidal dispersion in nano size consists of a solid lipid stabilized by surfactants that work in the interface area ${ }^{3}$. Some advantages of SLN, for instance the ability to protect the active compounds from degradation, controlled release, the ability to load a lipophilic or hydrophilic material, non-toxic and did not irritate $^{3}$. Limitations of SLN systems included loading capacity of the active ingredient was limited and the expulsion of active ingredient during storage. This was because of the high orderdness of the lattice crystal of solid lipid so that the active ingredient which has been entrapped easily squeezed out of the system. To overcome the limitations of the system SLN, a second-generation nanoparticle system that is nanostructured lipid carrier (NLC) was developed ${ }^{6}$. NLC are formed with both liquid and solid lipid as a blend mixture in such a ratio that they are solid at room temperature. The active ingridient can be incorporated in particle matrix in a molecular dispersed form or it can be arranged as amorphous cluster ${ }^{6}$.

The NLC manufacturing is done by mixing solid lipid in an amount greater than the liquid lipid. This research used solid lipid (Monostearin), liquid lipid (Miglyol 808) with a ratio of $6: 4,7: 3$, and $8 ; 2$, surfactant (Tween 80) and meloxicam as the active ingredient. Evaluation conducted in this study included physicochemical characteristics after strorage 40 days.

\section{MATERIALS AND METHODS}

Materials

The main materials used in this study is meloxicam (Apex Healthcare Limited), Monostearin (Sigma Aldrich), Miglyol 808 (Sigma Aldrich), Tween 80 (PT Croda), pro analysis Ethanol (Merck), Buffer component: $\mathrm{KH}_{2} \mathrm{PO}_{4}$ and $\mathrm{NaOH}$ pro analysis (Merck).

Methods

Preparation NLC System of Meloxicam

The Manufacturing of NLC meloxicam

This study used emulsification method (emulsification). Meloxicam-NLC system was made by melting the lipid phase (Monostearin and Miglyol 808 ) with different lipid ratios (6:4, 7: 3 and 8:2), and meloxicam at temperatures of $65^{\circ} \mathrm{C}$. At the same time, a solution of surfactant (Tween-80 and phosphate buffer $\mathrm{pH}$ 
Table 1: The formula of NLC meloxicam.

\begin{tabular}{llllll}
\hline Ingredient & Function & Concentrate & \multicolumn{3}{c}{ Concentrate (\% -w/ w) } \\
\cline { 4 - 6 } & & range $(\%)$ & Formulation I & Formulation II & Formulation III \\
\hline Meloxicam & Active ingredient & & 1 & 1 & 1 \\
Monostearin & Solid Lipid & $6^{*}$ & 6 & 7 & 8 \\
Miglyol 808 & Liquid Lipid & $3-4^{*}$ & 4 & 3 & 5 \\
Tween 80 & Surfactant & $1-15^{* *}$ & 5 & 5 & 5 \\
$\begin{array}{l}\text { Phosphate Buffer } \\
\text { pH 6 }\end{array}$ & & Until 100 & & \\
\hline
\end{tabular}

Table 2: The results of $\mathrm{pH}$ measurements of the meloxicam NLC system of formula 1, formula 2 and formula 3 on days 1,20 , and 40

\begin{tabular}{lll}
\hline Day & Formula & Average $\mathrm{pH} \pm \mathrm{SD}$ \\
\hline 1 & $2(7: 3)$ & $5.73 \pm 0.03$ \\
& $3(8: 2)$ & $5.75 \pm 0.01$ \\
20 & $1(6: 4)$ & $5.74 \pm 0.04$ \\
& $2(7: 3)$ & $5.74 \pm 0.04$ \\
40 & $3(8: 2)$ & $5.85 \pm 0.03$ \\
& $1(6: 4)$ & $5.83 \pm 0.02$ \\
& $2(7: 3)$ & $5.85 \pm 0.02$ \\
& $3(8: 2)$ & $5.87 \pm 0.02$ \\
\hline
\end{tabular}

$6.0 \pm 0.05$ ), was prepared and heated at a temperature of $65^{\circ} \mathrm{C}$ Hot surfactant solution then was dispersed into the hot lipid phase by using ultra-turax with a speed of 3400 rpm For 30 minutes. The next stage was cooling stage, then it was stirred using a magnetic stirrer at $100 \mathrm{rpm}$ until it Reached the temperature of $25^{\circ} \mathrm{C}$. The fixed NLC was measured to Determine the end weight of the $\mathrm{NLC}^{4,10}$. Evalution of the Physical Stability of NLC Meloxicam All NLC-MLX were evalution of physical stability before and after storage 40 days in their $\mathrm{pH}$, viscosity, particles size, and entrapment efficiency.

Determination of $\mathrm{pH}$

Determining $\mathrm{pH}$ of each test preparation was done using a $\mathrm{pH}$ meter.

Examination of the particle size and size distribution Examination of the particle size consisting of two treatments before and after UVB irradiated for 21 hours. Each sample was diluted to $200 \mathrm{ppm}$ stirring for 3 minutes at $500 \mathrm{rpm}$. Dilution checked by Delsa Nano Particle Size on the angle of 650 and a temperature of $25^{\circ} \mathrm{C}$. Data
Table 4 Measurement results of entrapment efficiency of meloxicam NLC system on days 1, 20, and 40

\begin{tabular}{lll}
\hline Day & Formula & Trapping Rate $(\%) \pm$ SD \\
\hline \multirow{2}{*}{1} & $2(7: 3)$ & $90.831 \pm 0.180$ \\
& $3(8: 2)$ & $94.358 \pm 0.109$ \\
& $1(6: 4)$ & $88.848 \pm 0.522$ \\
20 & $2(7: 3)$ & $91,561 \pm 0.412$ \\
& $3(8: 2)$ & $95,703 \pm 0,510$ \\
& $1(6: 4)$ & $90.996 \pm 0.440$ \\
40 & $2(7: 3)$ & $94.343 \pm 0.168$ \\
& $3(8: 2)$ & $95,850 \pm 0.535$ \\
\hline
\end{tabular}

obtained were average particle diameter and Polydispersity Index (PI). All measurement was replicated 3 times.

Percentage of Drug Entrapment (Loading capacity)

Drug entrapment efficiency was determined by centrifugation method. It was calculated from the ratio of the medicinal amount in NLC to total added drug amount. Centrifugation was carried out using Hettich Rotofix 32 Centrifuge. About 1 gm of NLC dispersion containing the drug was placed in the centrifuge tube, and sample were centrifuged at 2,500 rpm for 45 minutes. The amount of the free drug in the supernatant was estimated at $362 \mathrm{~nm}$ by spectrophotometry.

\section{RESULT AND DISCUSSION}

Determination of $\mathrm{pH}$ after storage

The $\mathrm{pH}$-measurement results of each of the formulas after storage for 40 days can be seen in Table 2. Based on the results of statistical analysis ANOVA two way on $\mathrm{pH}$ testing NLC meloxicam on day 1, day 20 and day 40 showed that there was no difference in $\mathrm{pH}$ change significantly during storage, as shown by $\mathrm{F}$ value of 0,579

Table 3 The results of particle size measurements and polypspersity index of NLC meloxicam system of formula 1, formula 2, and formula 3 on days 1, 20, and 40

\begin{tabular}{llll}
\hline Day & Formula & Particle Size $(\mathrm{nm}) \pm$ SD & Polidispersitas Index \pm SD \\
\hline \multirow{3}{*}{1} & $1(6: 4)$ & $518.9 \pm 291.3$ & $0.299 \pm 0.236$ \\
& $2(7: 3)$ & $472.1 \pm 107.1$ & $0.616 \pm 0.092$ \\
20 & $3(8: 2)$ & $421.9 \pm 137.1$ & $421.9 \pm 137.1$ \\
& $1(6: 4)$ & $244.2 \pm 23.2$ & $0.438 \pm 0.054$ \\
& $2(7: 3)$ & $235.4 \pm 41.6$ & $0.650 \pm 0.251$ \\
40 & $3(8: 2)$ & $416.3 \pm 143.4$ & $0.495 \pm 0.155$ \\
& $1(6: 4)$ & $333.9 \pm 104.0$ & $0.474 \pm 0.097$ \\
& $2(7: 3)$ & $404.0 \pm 240.8$ & $0.565 \pm 0.171$ \\
\hline
\end{tabular}


and significant against $\alpha=0.05(0.571)$. The $\mathrm{p}$ value for the formula* day is 0.958 , it can be concluded that there is no significant $\mathrm{pH}$ difference between formula 1 , formula 2 and formula 3 on storage time (days). After 40 days of storage it can be concluded that the meloxicam NLC system formed shows the stability of the Meloxicam NLC system in the absence of $\mathrm{pH}$ change.

Examination of the particle size and size distribution

The result of measuring the particle size of each formula after storage for 40 days can be seen in Table 3. Based on the results of two-way ANOVA statistical analysis particle size measurements obtained $F$ value of 2.861 and significant to $\alpha=0.05(0.083)$. The $\mathrm{p}$ value for the formula* day is 0.390 , it can be concluded that there is no significant particle size difference between formula 1 , formula 2, and formula 3 on storage time (days). This shows that there is no significant difference in particle size of formula 1, formula 2, and formula 3 during storage from Day 1, 20th, and 40th. After 40 days of storage it can be concluded that the meloxicam NLC system formed shows the stability of the system NLC in the absence of particle size changes.

Measurement of Drug Entrapment efficiency After Storage 40 Days

The measurement of trapping efficiency of each of the formulas after storage for 40 days can be seen in Table 4 . Based on the results of two-way ANOVA statistical analysis trapping efficiency measurements obtained $\mathrm{F}$ value of 113.06 and significant to $\alpha=0.05$ (0.000), demonstrating the difference between the trapping efficiency of the storage time the 1st, 20th, and to- 40. This difference in the efficiency of the trapping is indicated by the increase in trap efficiency after 40 days of storage, indicating that the meloxicam NLC system formed remains stable with no reduction in trap efficiency.

\section{CONCLUSION}

NLC system of meloxicam using lipid Monostearin and Miglyol 808 ratio of 8: 2 is the most optimal combination, because it shows physicochemical characteristics according to the criteria of NLC system of meloxicam the post 40-day-storage.

\section{CONFLICT OF INTEREST}

I hereby state that no conflict of interest exists between me and the co-author of this manuscript.

\section{REFERENCES}

1. Bertram, G and Katzung MD., 2009. Basic and Clinical Pharmacology, $11^{\text {th }}$ Ed. United States: McGraw-Hill Companies Ins., pp.817-819

2. Cirri, M., Bragagni, M., Mennini, P., 2012. Development of a New Delivery System Consisting "Drug-in Cyclodextrin-in Nanostructured Lipid Carriers" for Ketoprofen Topical Delivery. Eur $J$ of Pharm and Biopharm 80 (1): 46-53.

3. Ekambaran, P., Shatali A. A. H., Priyanka K, 2011. Solid Lipid Nanoparticle: A Review. Scientific Review and Chemical Communication. Vol.2, pp.80-102

4. Khurana, S., Jain, NK, Bedi, PMS, 2013. Development and Characterization of a Novel Controlled Release Drug Delivery System Based on Nanostructured Lipid Carriers Gel for Meloxicam. Life Sci 93: 763-772.

5. Muller, RH, Mader, K., Gohla, S., 2002. Solid Lipid Nanoparticles for Controlled Drug Delivery a Review of the State of the Art. Eur $J$ of Pharm and Biopharm 50: 161-177.

6. Pritu Pathak, A.K. Phatak, 2015, Preparation and Evaluation of Nanostructured Lipid Carriers Evaluation of Nanostructured Lipid Carriers of Azelaic Acid in Topical Formulation (Hydrogel), International Journal of Current Pharmaceutical Review and Review and Research; 6(3); 188-194.

7. Montenegro, Lucia, 2014, Nanocarriers For Skin Delivery of Cosmetic Antioxidant, Journal of Pharmacy and Pharmacognosy Research, Vol 2, No.4, pp 73-92.

8. Souto, EB, and Muller, RH, 2007. Lipid Nanoparticles (Solid Lipid Nanoparticles and Nanostructured Lipid Carriers) for Cosmetic, Dermal, and Transdermal Applications. Drug and Pharm Sci 166: 213-232.

9. Westesen, K., Siekmann, B., 1997. Investigation of the Gel Formation of Solid Lipid Nanoparticles StabilizedPhospholipids. Inter J of Pharm 151: 35-45.

10. Yuan, H., Wang LL, Du YZ, You, J., Hu, FQ, Zeng, S., 2007. Preparation and Characteristics of Nanostructured Lipid Carriers for Control Releasing Progesterone by Melt Emulsification. Colloids and Surfaces Biointerfaces 60 (2): 174-179. 